\title{
Informing the Design of an Authoring Tool for Developing Social Stories
}

\author{
Aurora Constantin ${ }^{1, *}$, Helen Pain ${ }^{1}$, and Annalu Waller ${ }^{2}$ \\ ${ }^{1}$ University of Edinburgh, School of Informatics, Crichton St 10, \\ Edinburgh, EH8 9AB UK \\ A.Constantin-2@sms.ed.ac.uk, helen@inf.ed.ac.uk \\ ${ }^{2}$ University of Dundee, School of Computing, Perth Road, \\ Dundee, DD1 4HD UK \\ awaller@computing. dundee.uk
}

\begin{abstract}
This paper describes the design of an authoring tool, ISISS (Improving Social Interaction through Social Stories), for supporting practitioners using social stories to enhance social interaction in children with Autism Spectrum Conditions (ASC). The goals of the research are: 1) to determine practitioners' current procedures when working with social stories; 2) to discover how technology can better support such practitioners in the development and use of social stories that focus on improving children's social communication skills. An exploratory study was conducted with experienced practitioners, resulting in a number of design principles. Two low-functioning prototypes were developed, and explored in a second study. Further work is discussed.
\end{abstract}

Keywords: Paper-based Interfaces, Design, Autism, ASC, Educational Tool, Assistive Technology for Children with ASC, Authoring Tool.

\section{Introduction}

Children with Autistic Spectrum Conditions (ASC) frequently have difficulties with social interaction [19]. These can be addressed through the use of social stories. The concept of social story was devised by Carol Gray as "a short story-defined by specific characteristics - that describes a situation, concept, or social skill using a format that is meaningful for people with ASD" [7]. It is written from the student's point of view and is a guide to follow when they have difficulties with a social situation (see Fig. 1). They are used to help the child acquire appropriate behaviour, reduce inappropriate behaviour, teach routines, teach skills, or cope with transitions and novel situations. Meta-reviews of social story interventions $[11,16]$ focus on their effectiveness and on the impact of variables such as goal addressed, length of intervention, or participant characteristics. They conclude that social stories are promising, though it is not clear what variables are crucial to their efficacy.

\footnotetext{
* Corresponding author. 
The aims of this research are: 1) to determine practitioners' procedures when working with social stories, and 2) to discover how technology can better support such practitioners. The longer term goal is to evaluate if computer-based technology can support and enhance the writing, presenting and assessment of social stories.

Taking my temperature
Sometimes if I am sick I get very hot.
The doctor needs to know how hot I am.
He will take my temperature with a thermometer.
He will put the thermometer in my ear. This will not hurt.
I might hear a beep or click noise, and this will not hurt.
The doctor can see if I am sick. Then he can help me get better.
The doctor will be happy and mum will be happy.

Fig. 1. Example of social story

Section 2 of this paper presents a short review of social stories and their importance for children with ASC. Section 3 describes a study which aimed to uncover practitioners' procedures and practices in order to see what support they need. The implications for design are considered in section 4. Section 5 assesses current tools for social stories in relation to the design principles and requirements elicited from the study. The design of an authoring tool to support practitioners in developing social stories is proposed. Section 6 presents the formative evaluation of the initial prototypes. Section 7 includes conclusions and future work.

\section{Background}

Psychological research has identified three dominant cognitive theories of autism: theory of mind, weak central coherence, and executive dysfunction [15]. Individuals with ASC frequently have impaired Theory of Mind: they have difficulty understanding what other people think, feel, or intend to do [2, 6, 13]. A social story can provide information about how other people think and behave in a specific social situation, using perspective sentences that refer to other people's thoughts, feelings, actions and motivation. In this way a social story might reduce or remove the confusion and ameliorate the deficit in Theory of Mind.

The weak central coherence theory describes the inability to build higher level meaning. An individual with ASC may be too focused on detail and miss the 'whole picture' $[9,15]$. Social stories are helpful in guiding individuals with ASC to identify the relevant details for a specific event and to correct mistaken assumptions; providing logical connections can help the individual to grasp the bigger picture.

Executive Function is a generic term for functions such as: initiating, sustaining, shifting and inhibition actions [24]. It is thought to be responsible for handling novel situations where the routine is insufficient for optimal performance [15]. Social 
stories seem to be useful in reducing or removing the deficit in executive functions, by providing an individual with ASC with planning and organizing strategies in specific social situations, with initiation and impulse control.

Gray [8] suggests that social stories (SS) should be customized to meet the distinct needs and skills of the child, such as reading and comprehension skills, learning style, interests and attention. She provides criteria and guidelines to support the development of SS, including use of the first person; sharing social information in a simple, literal way; answering "wh" questions (who is doing, what, where, when and why?) and how questions, and use of positive language, with reference to positive rather than negative behaviour. Illustrations should be used when appropriate. She defines different sentence types to be used, including descriptive sentences (factual statements); perspective (describing a person's internal state, thoughts, feelings, beliefs); cooperative (identifying what others will do to help); directive (identifying a suggested response or a choice to a situation); affirmative (enhancing the meaning of previous statements), and control sentences (identifying strategies for recalling or applying information in SS). Descriptive, directive, perspective and control sentences may be either complete or partial (e.g. "Mom and Dad will feel if I finish all my dinner"). She recommends a ratio between sentences of $0-1$ directive and control to 2 or more descriptive, perspective, affirmative and cooperative.

\section{Exploring How Practitioners Develop Social Stories}

The approach taken in this research is primarily a Participatory Design approach, with practitioners as the main stakeholders. A study was conducted to better understand the following: 1) the process of developing SS; 2) the challenges encountered by practitioners in doing so; 3 ) to collect examples of SS developed, and typical content; 4) the tools currently used to support social story development. This study involved 4 practitioners who worked in special schools and had considerable experience in developing social story interventions for children with ASC. The practitioners were invited to participate in the study, and informed of its purpose. They were asked to think about their procedures and practices in SS, in advance of participation. The 2 hour study session took place outside of the school setting.

Each practitioner was asked to write a social story. Practitioners were invited to express their thoughts aloud ('think aloud' protocol) while building the social story. Following this, semi-structured interviews were used to better understand the practitioners' experience and challenges when using SS with children with ASC. Both activities were recorded and video transcripts analysed qualitatively, using open, axial and theoretical coding [17] to build a conceptual framework of social story intervention. Further literature was reviewed and incorporated into the framework. The results suggested that practitioners would value support for developing SS using a specialised computer tool, as an alternative to pen-and-paper or word processing methods currently used. The outcomes led to design principles and initial requirements for a 'social stories authoring tool', described below. 


\section{Supporting Practitioners in Developing Social Stories}

The study revealed that a tool for supporting practitioners in developing SS might help them organise their work, and address the main challenges they face, including re-using resources developed based on individuals' profiles. Design should also consider Gray's guidelines and best practice. The steps that practitioners use are identified and elaborated, along with other core concepts, in Fig. 2: the exterior arrows suggest that challenges and content are determined by steps and goals, while the interior arrows suggest that the four concepts are part of social story intervention. These led to functional requirements including being able to create new stories; extend and edit existing ones; browse and search a library of stories and related images; annotate story sentences; present them in various forms; create and edit child profiles; manage social story development through the use of status display, reminders, etc. and to record progress through and manage assessment of SS.

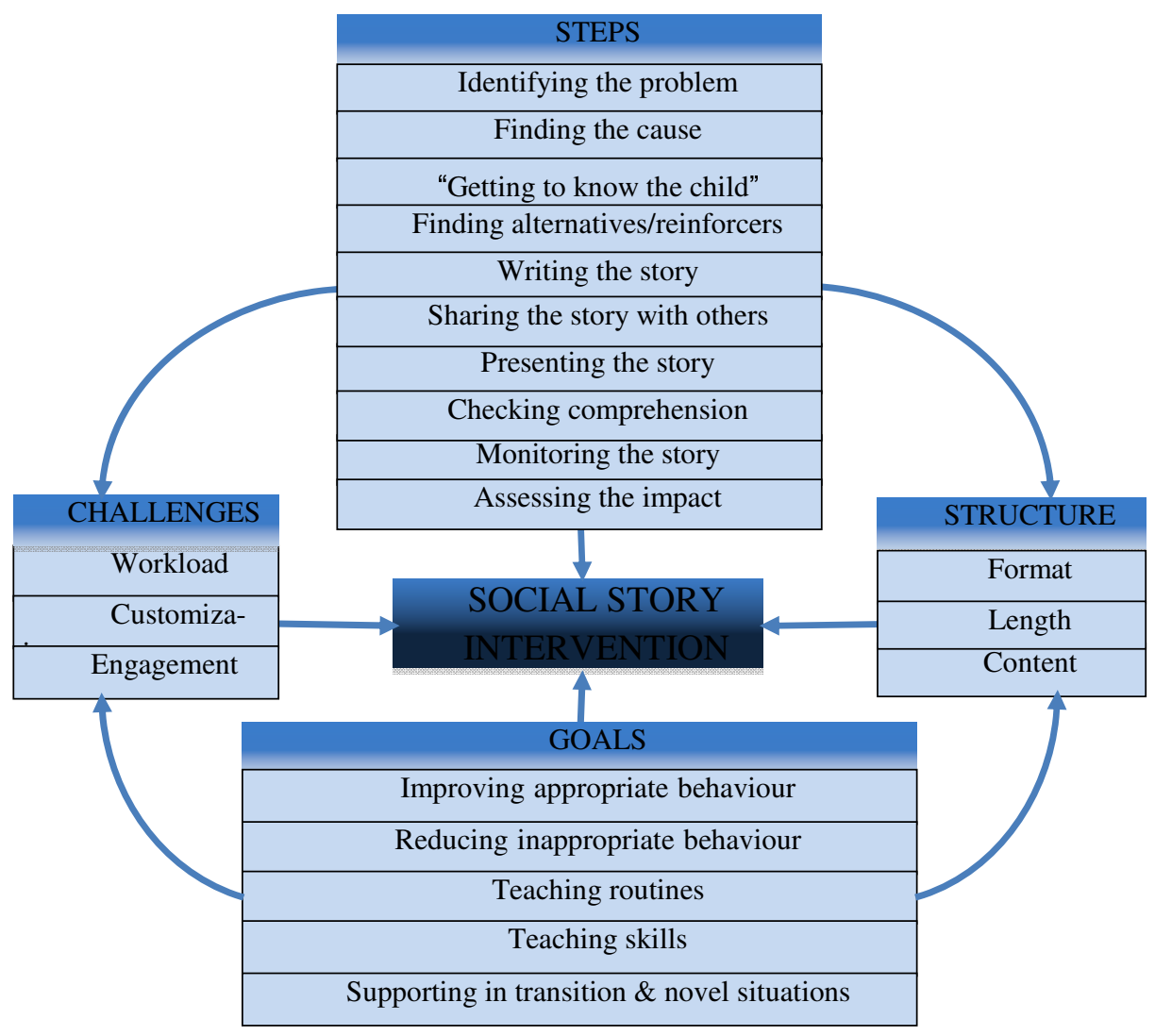

Fig. 2. The conceptual framework of social story intervention 
A number of challenges were identified, and these are incorporated in design principles to guide the design of tools to support social story development.

Ease the Practitioners' Workload. A major challenge that practitioners encounter is the time spent in preparing educational materials. Although SS seem to be less demanding than other educational strategies, the whole process of preparing, writing, presenting and assessing a social story is labour intensive. To address these problems, any support tool needs to be simple, intuitive and must help practitioners organise their work, and support the steps in the development process. The practitioners identified that the tool should allow them to reuse SS, symbols and photographs and to monitor the impact of SS on children. Data about the child's progress should be accessed and presented in various ways, enabling practitioners to get new insights into the child's behaviour and assess the success of the social story.

Design for Customizability. A common desire is to quickly customize newly created or re-used SS. Users should be allowed to create resources for each child, to add their own SS, symbols, photographs, rewards, as well as interests and strengths (which should be accessible from the child's profile). The stories should also be customizable to the story topic content, be re-usable and sharable with other practitioners and children. Text to speech capabilities should be added to accommodate children with reading difficulties. Options to choose between various layouts should be provided.

Design for Engagement. This could be met by customization to the child's interests and familiar context (e.g. images of familiar people). Practitioners could add rewards at the end of SS (e.g. animated characters, songs) adapted to each particular child. Social stories with partial sentences check comprehension, but may also make the system more interactive, potentially improving the child's engagement with the tool.

\section{Current Tools for Social Stories}

Applications for Creating Social Stories. e.g. Story Builder [21], Story2Learn [22], Sandbox Learning [18], Pictello [14], Social Stories [20], and StoryMaker [23], were considered in relation to the need to support the steps proposed for story development, and the design principles and requirements, as above. These applications focus primarily on building, editing and presenting SS, but do not generally support checking the comprehension, monitoring the story, or assessing SS. None of the applications provides an option to annotate the type of sentences, as Gray defines them. The applications are presented as allowing customisation, but this consists largely of changing font sizes and colours, background and the type of voice (when using text to speech technology, e.g. Pictello). None permits change of the story layout, nor provide the option to create resources for a particular child (e.g. favourite pictures, symbols or rewards) or for their reuse when creating new stories for that child. None permit the creation of a profile of the child, or storing information about the SS created, or their progress. 
The limitations identified means these applications are not viable as the basis for a computer-based application to support practitioners in developing SS. Anecdotal evidence shows that practitioners sometimes use generic tools to create SS: Communicate: In Print [5], Boardmaker [3], or Comic Life [4], though these do not satisfy the requirements. This research suggests that, to better support practitioners, research is needed to investigate whether a computer based tool could be developed that satisfies more of the requirements and principles identified through studies with practitioners.

Authoring Tools. enable users to author material using an intuitive interface. In his analysis of the state of art of authoring intelligent tutoring systems (ITS), Murray [12] argues that authoring tools: a) reduce the effort used in creating ITS; b) reduce the necessary skill threshold for developing ITS; c) support the author in articulating or organizing her work; d) scaffold good design principles; e) facilitate rapid prototyping. Many of these arguments agree with the principles and requirements defined above, and support the use of an authoring tool to help practitioners in their work, enabling rapid customization, flexibility and requiring no programming skills.

\section{Formative Evaluation of Initial Prototypes}

A second study was conducted with practitioners. The goals of this study were: 1) to explore design alternatives; 2) to discover usability problems and solutions to overcome them; 3) to generate new ideas to include into the systems' features and interaction, and 4) to refine the system specification. Two versions of low-functioning prototypes were created using Balsamiq Mockups [1], according to the requirements and design principles (see screenshots examples in Fig 3: a \& b). They were explored with ten practitioners having experience in using SS with children with ASC. In each two-hour session constructive interaction was applied, a version of the thinking aloud protocol which requires two practitioners at a time [10]. The practitioners were presented with four scenarios and invited to follow each scenario using each prototype, by pressing on buttons and menus, and simulating typing. A researcher playing the "computer" role changed the screens according to the users' actions. The main researcher, acting as a mediator, encouraged the practitioners to express their opinions and preferences for various features of the prototypes. Brainstorming was then used to find solutions for the problems encountered and to generate new ideas. Qualitative analysis of session recordings was used to determine practitioners' preferences, usability problems and solutions, and to extract new ideas for design.

The problems found were categorized into five groups: 1) page layout; 2) navigation/workflow; 3) concepts and terminology; 4) content, and 5) functionality. Based on these, the system interfaces and specification were refined.

Figure 3 shows the revised working prototype. The tab navigator containing pictures, symbols, layouts and resources (for a child) was preferred on the left side (Fig.3:d). The navigation buttons were placed in left and right bottom corners of the story panel. The option to annotate the sentences was considered of high importance for practitioners and included in the working prototype (Fig.3:e). 




Fig. 3. The Prototypes: a) initial prototype 1; b) initial prototype 2; c) working prototype; d) tab navigator for customization; e) annotate sentences; f) navigation button

\section{$7 \quad$ Discussion and Future Work}

This paper reports on research that informs the design of an authoring tool for writing, presenting and assessing SS for children with ASC. This research has been guided by the methods of Participatory Design. The first study uncovered practitioners' procedures when developing SS. The focus was on the core concepts and themes that could be translated into design principles and system requirements. Although practitioners were aware that tools for social story interventions exist, most of them preferred paper and pencil to write SS, existing tools being either too complex to use or not flexible enough. They sometimes use tools not specific for SS, in order to access the library of symbols (in [5] and [3]) and because of the simplicity of the interface (in [4]). Through the second study the requirements were clarified, different design strategies explored, and interfaces and specifications refined. In both studies the practitioners contributed in creating knowledge and showed interest and enthusiasm for the project.

These are the first steps in a project aimed at better scaffolding for practitioners in developing SS. In turn this may increase the positive impact of SS on social communication skills of children with ASC. The results have been incorporated in an evolutionary prototype authoring tool, which will be further evaluated with practitioners.

\section{References}

1. Balsamiq Mockups, http: / / www . balsamiq.com/

2. Baron-Cohen, S., Leslie, A.M., Frith, U.: Does the autistic-child have a theory of mind? Cognition 21(1), 37-46 (1985) 
3. Boardmaker, http://www. mayer-johnson.co.uk/what-is-boardmaker/

4. Comic Life, http: / /www. comiclife.com/

5. Communicate: In Print, http://www.widgit.com/products/inprint/ index.htm

6. Frith, U.: Autism: Explaining the enigma. Blackwell, Oxford (1989)

7. Gray, C.: Social Stories (2003), http: / / www . thegraycenter .org/

8. Gray, C.: Social Stories 10.0. Jenison Autism Journal 15(4), 2-21 (2004)

9. Happé, F., Frith, U.: The weak coherence account: detail-focused cognitive style in autism spectrum disorders. Journal of Autism and Developmental Disorders 36(1), 5-25 (2006)

10. Holzinger, A.: Usability engineering for software developers. Communications of the ACM 48(1), 71-74 (2005)

11. Kokina, A., Kern, L.: Social Story interventions for students with autism spectrum disorders: A meta-analysis. Journal of Autism and Developmental Disorders 40(7), 812-826 (2010)

12. Murray, T.: Authoring Intelligent Tutoring Systems: An Analysis of the State of the Art. International Journal of Artificial Intelligence in Education 10, 98-129 (1999)

13. Perner, J., Lang, B., Kloo, D.: Theory of mind and self-control: more than a common problem of inhibition. Child Development 73(3), 752-767 (2002)

14. Pictello, http://www.assistiveware.com/product/pictello

15. Rajendran, G., Mitchell, P.: Cognitive theories of autism. Developmental Review 27(2), 224-260 (2007)

16. Reynhout, G., Carter, M.: Social Stories ${ }^{\mathrm{TM}}$ for children with disabilities. Journal of Autism and Developmental Disorders 36(4), 445-469 (2006)

17. Saldana, J.: The Coding Manual for Qualitative Researchers. Sage Publications, London (2009)

18. Sandbox Learning, http: / / www . sandbox-learning . com

19. Shattuck, P.T., Seltzer, M.M., Greenberg, J.S., Orsmond, G.I., Bolt, D., et al.: Change in autism symptoms and maladaptive behaviours in adolescents and adults with an autism spectrum disorder. J. Autism Dev. Disord. 37, 1735-1747 (2007)

20. Social Stories, http://a4cwsn.com/2012/09/social-stories/

21. Story Builder, http://www. leedsmet.ac.uk/inn/usabilityservices/ download2.htm

22. Story2Learn, https://itunes.apple.com/gb/app/stories2learn/ id3 48576875 ? $\mathrm{mt}=8$

23. StoryMaker, http://udm4.com/iPhone/StoryMaker_for_Socia-3170588

24. Zelazo, P.D., Müller, U.: Executive function in typical and atypical development. In: Goswami, U. (ed.) Handbook of Childhood Cognitive Development. Blackwell, Oxford (2002) 